University of Wollongong

Research Online

Faculty of Engineering and Information

Faculty of Engineering and Information

Sciences - Papers: Part A

Sciences

$1-1-2016$

\title{
Characterisation of a cobalt- 60 small-beam animal irradiator using a realtime silicon pixelated detector
}

C Porumb

University of Wollongong, csp528@uowmail.edu.au

Jocelyn Davies

Australian Nuclear Science And Technology Organisation

Vladimir Perevertaylo

SPA BIT

Anatoly B. Rosenfeld

University of Wollongong, anatoly@uow.edu.au

Marco Petasecca

University of Wollongong, marcop@uow.edu.au

Follow this and additional works at: https://ro.uow.edu.au/eispapers

Part of the Engineering Commons, and the Science and Technology Studies Commons

Research Online is the open access institutional repository for the University of Wollongong. For further information contact the UOW Library: research-pubs@uow.edu.au 


\title{
Characterisation of a cobalt- 60 small-beam animal irradiator using a realtime silicon pixelated detector
}

\author{
Abstract \\ The paper presents a study performed by the Centre for Medical Radiation Physics (CMRP) using a high \\ spatial and temporal resolution silicon pixelated detector named MagicPlate- 512 . The study focuses on \\ the characterisation of three pencil beams from a low-dose rate, $6 \mathrm{TBq}$, cobalt- 60 source, in terms of \\ percentage depth dose, beam profiles, output factor and shutter timing. Where applicable, the findings \\ were verified against radiochromic EBT3 film and ionization chambers. It was found that the results of the \\ MagicPlate-512 and film agreed within $0.9 \mathrm{~mm}$ for penumbra and full-width at half-maximum \\ measurements of the beam profiles, and within $0.75 \%$ for percentage depth dose study. The dose rate of \\ the cobalt-60 source was determined to be $(10.65 \pm 0.03) \mathrm{cGy} / \mathrm{min}$ at $1.5 \mathrm{~cm}$ depth in Solid Water. A \\ significant asymmetry of the small pencil beam profile was found, which is due to the irregular machining \\ of the small collimator. The average source shutter speed was calculated to be $26 \mathrm{~cm} / \mathrm{s}$. The study \\ demonstrates that the MagicPlate-512 dosimetry system, developed at CMRP, is capable of beam \\ characterisation even in cases of very low dose rate sources.
}

\section{Keywords}

cobalt, detector, silicon, realtime, irradiator, characterisation, animal, pixelated, beam, small, 60

\section{Disciplines}

Engineering | Science and Technology Studies

\section{Publication Details}

Porumb, C. S., Davies, J. B., Perevertaylo, V., Rosenfeld, A. B. \& Petasecca, M. (2016). Characterisation of a cobalt- 60 small-beam animal irradiator using a realtime silicon pixelated detector. Journal of Instrumentation, 11 (4), P04014-1-P04014-14. 


\title{
Characterisation of a cobalt-60 small-beam animal irradiator using a realtime silicon pixelated detector
}

\author{
Claudiu S. Porumb ${ }^{a}$, Justin B. Davies ${ }^{b}$, Vladimir Perevertaylo ${ }^{c}$, Anatoly B. \\ Rosenfeld ${ }^{\mathrm{a}}$, and Marco Petasecca ${ }^{\mathrm{a}}$, \\ ${ }^{a}$ Centre for Medical Radiation Physics, \\ University of Wollongong, Northfields Avenue, Gwynneville, New South Wales, Australia \\ ${ }^{b}$ Australian Nuclear Science and Technology Organisation, \\ Lucas Heights, New South Wales, Australia \\ ${ }^{c}$ SPA-BIT, \\ Kiev, Ukraine \\ E-mail: csp528@uow. edu. au
}

ABSTRACT: The paper presents a study performed by the Centre for Medical Radiation Physics (CMRP) using a high spatial and temporal resolution silicon pixelated detector named MagicPlate-512. The study focuses on the characterisation of three pencil beams from a lowdose rate, $6 \mathrm{TBq}$, cobalt- 60 source, in terms of percentage depth dose, beam profiles, output factor and shutter timing. Where applicable, the findings were verified against radiochromic EBT3 film and ionization chambers. It was found that the results of the MagicPlate-512 and film agreed within $0.9 \mathrm{~mm}$ for penumbra and full-width at half-maximum measurements of the beam profiles, and within $0.75 \%$ for percentage depth dose study. The dose rate of the cobalt- 60 source was determined to be $(10.65 \pm 0.03) \mathrm{cGy} / \mathrm{min}$ at $1.5 \mathrm{~cm}$ depth in Solid Water. A significant asymmetry of the small pencil beam profile was found, which is due to the irregular machining of the small collimator. The average source shutter speed was calculated to be $(65 \pm$ 8) $\mathrm{cm} / \mathrm{s}$. The study demonstrates that the MagicPlate-512 dosimetry system, developed at CMRP, is capable of beam characterisation even in cases of very low dose rate sources.

KEYWORDS: Dosimetry concepts and apparatus; Solid state detectors; Electronic detector readout concepts. 


\section{Contents}

1. Introduction $\quad 1$

2. Materials and methods $\quad 2$

2.1 MagicPlate-512 2

2.2 Readout system $\quad 2$

2.3 Ionisation chamber $\quad 3$

2.4 Film 3

2.5 Dose calibration and equalization procedures 4

$\begin{array}{ll}2.6 \text { Percentage depth dose } & 5\end{array}$

$\begin{array}{ll}2.7 \text { Beam output factors } & 5\end{array}$

$\begin{array}{ll}2.8 \text { Beam profiles } & 6\end{array}$

$\begin{array}{ll}2.9 \text { Shutter timing study } & 6\end{array}$

$\begin{array}{ll}\text { 3. Results and discussion } & \mathbf{7}\end{array}$

$\begin{array}{ll}3.1 \text { Percentage depth dose } & 7\end{array}$

$\begin{array}{ll}3.2 \text { Beam profiles } & 8\end{array}$

$\begin{array}{lr}3.3 \text { Beam output factors } & 10\end{array}$

$\begin{array}{ll}3.4 \text { Shutter timing study } & 11\end{array}$

4. Conclusion $\quad 12$

\section{Introduction}

The use of animal research in treatment and radiotherapy can help advance our understanding of physical and biological processes occurring in-vivo and can potentially increase the effectiveness of treatment of disease in humans, leading to an increased quality of life [1-4]. In this paper, the characterisation of an Atomic Energy of Canada Limited (AECL) Theratron cobalt-60 low-dose irradiator used for small animal and cell irradiation is presented. The irradiator is equipped with three lead collimators to shape the gamma-ray beam to circles of approximately 5, 10 and $20 \mathrm{~mm}$ in diameter. The irradiator is also provided with a fast mechanical shutter. The pencil beams are intended for the irradiation of the head of an animal (mouse or rat) whilst collimator is shielding the remaining of its body, with the intention to study the effect of ionising radiation on nervous system function. Because of the size of the target volume, it is thus important to estimate the shape of the delivered dose accurately and identify the irregularities in the profiles, if any. There was a need to substantially improve the characterisation of the radiation fields previously performed with an ionisation chamber, as the volume averaging effect of the ionisation chamber sensitive volume prevented the accurate determination of high dose gradient profiles realised by the means of the small radius collimators. Use of radiochromic film is recommendable for their intrinsic spatial resolution for 
the profiles but cannot characterise the performance of the mechanical beam shutter and the percentage of dose delivered during the beam-on/beam-off transient.

The percentage depth dose, beam output factors, and beam profiles are presented, as well as the timing study for the source's shutter using a real-time semiconductor detector array.

\section{Materials and methods}

In this study, the MagicPlate-512 (MP512) detector developed by Centre for Medical Radiation Physics (CMRP - University of Wollongong - Australia) was used for the real-time acquisition, alongside a calibrated ionisation chamber [5] and radiochromic film [6],[7], to characterise a Theratron, $6 \mathrm{TBq}, \mathrm{Co}-60$ gamma irradiator equipped with three collimators of diameter approximately 5,10 and $20 \mathrm{~mm}$. The MP512 system is comprised of three main parts: pixelated radiation detector, the detector readout electronics and the control software.
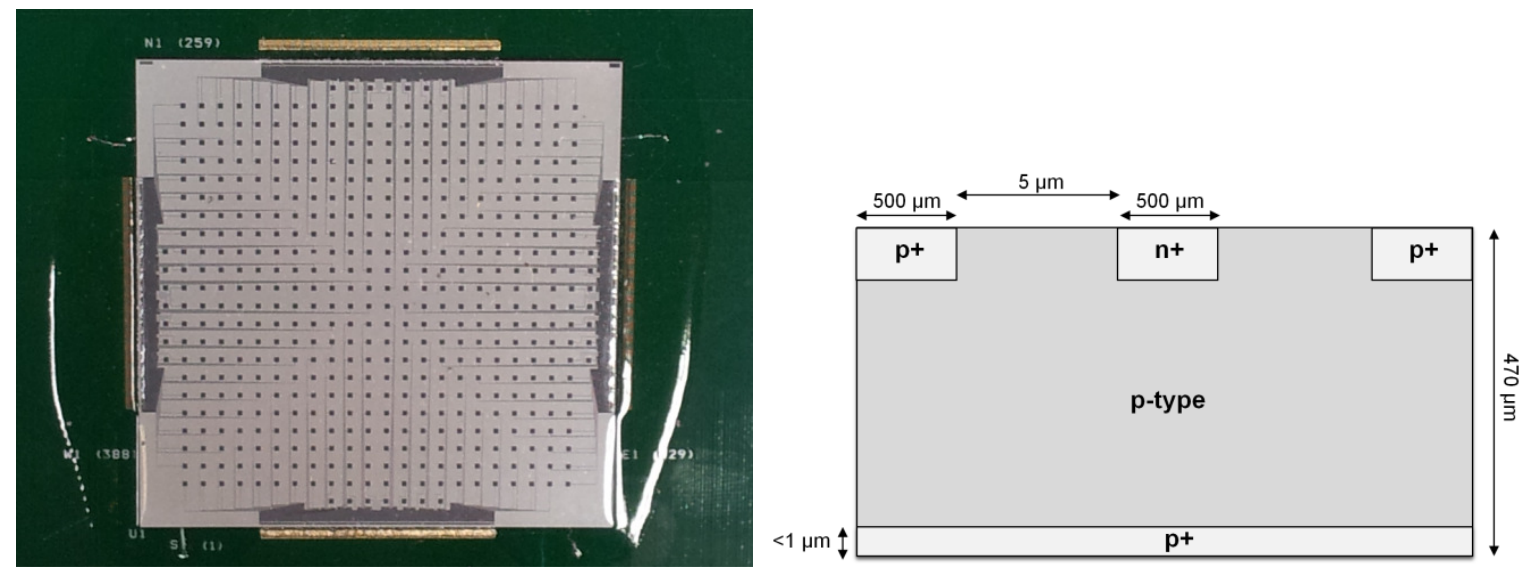

Figure 1: (left) MagicPlate-512 detector array mounted on the PCB; (right) simplified schematic diagram of the single pixel structure (not to scale)

\subsection{MagicPlate-512}

The silicon detector used in this study is the MP512 detector. It is a monolithic 2D array of 512 diodes, implanted on a p-type silicon substrate (figure 1), designed by CMRP and manufactured at the microelectronics foundry by SPA-BIT. Each pixel element is a planar structure implanted on $470 \mu \mathrm{m}$ thick p-Si substrate with an $\mathrm{n}^{+}$core of $0.5 \times 0.5 \mathrm{~mm}^{2}$, surrounded by a uniform $\mathrm{p}^{+}$implant ( $\mathrm{p}$-stop). The back side of the detector has a similar $\mathrm{p}^{+}$implant to realize the ohmic back contact and is polarised at the same potential of the front side diode (passive mode). The MP512 silicon detector has a total area of $52 \times 52 \mathrm{~mm}^{2}$ and pixel pitch of 2 $\mathrm{mm}$. The array is wire bonded to a thin $(500 \mu \mathrm{m})$ printed circuit board (PCB) which provides the fan-out for connection of the sensor to the readout electronics. Further details of MP512 and its performance as small field dosimeter for external beam radiotherapy can be found in ref. [8].

\subsection{Readout system}

The electronic readout is based on the 64-channel Texas Instruments AFE0064 analog-todigital converter (ADC) chip. Eight chips comprise the detector readout, which are controlled by a Xilinx Spartan 3 field-programmable gate array (FPGA). The FPGA module features a standard USB 2.0 connection, and provides the interface between the readout electronics and the data acquisition computer. The software used for controlling the acquisition is a dedicated 
graphical user interface program developed in-house at CMRP. The program is named Romulus Radiation Tools; it is a multi-threaded C++ software application dedicated to real-time 2D and 3D visualisation of CMRP detectors, and off-line statistical analysis of their response. The electronic readout system has been characterised by Fuduli et al. in a separate study [9].

In this study, the readout system was set to acquire using a $2000 \mu$ s integration window, at a repetition rate of $200 \mathrm{~Hz}$. Each pixel was set a maximum charge collection of $0.5 \mathrm{pC}$ per frame. The maximum charge setting was divided in a 16-bit number; this is referred to as the dynamic range.

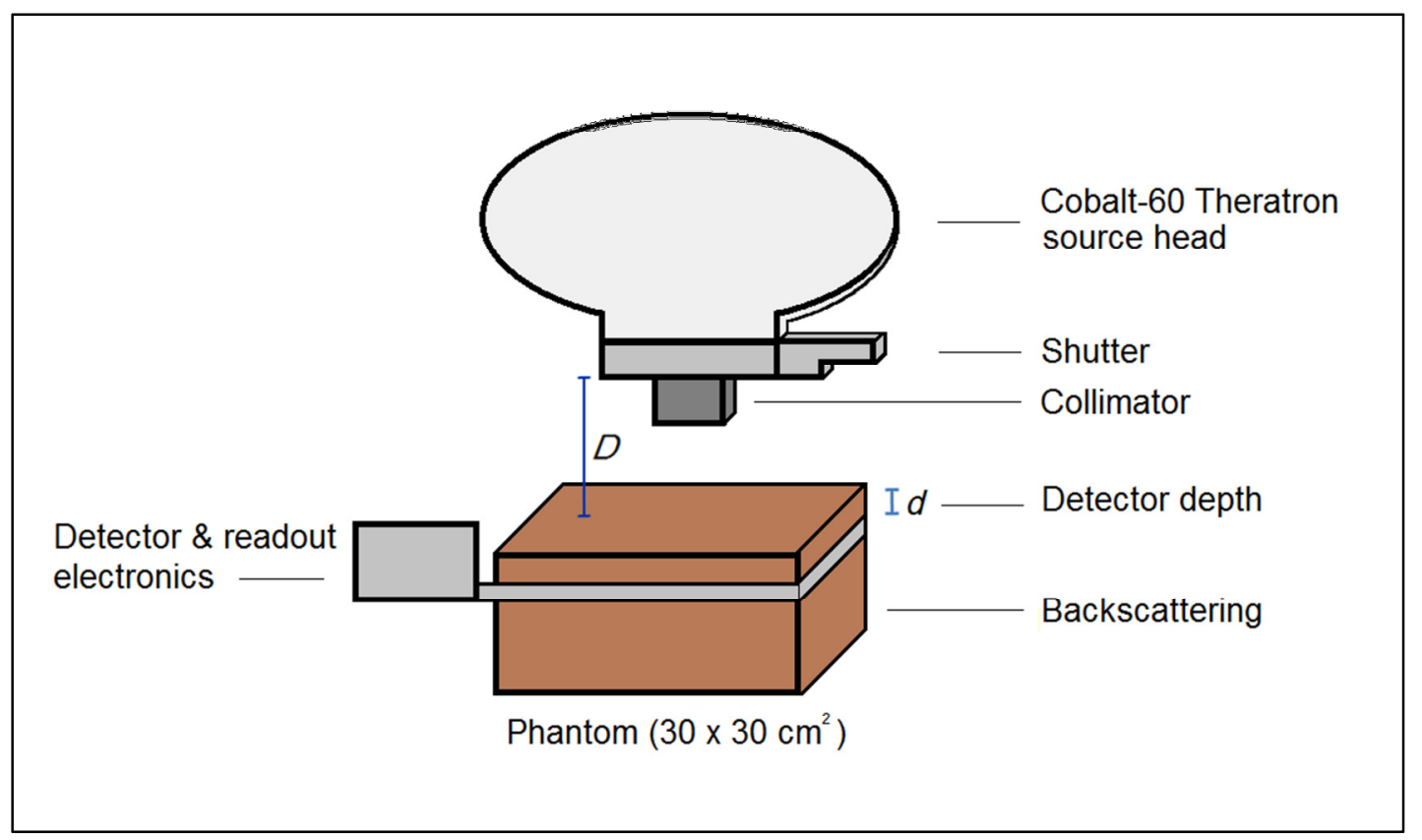

Figure 2: Schematic diagram of experimental setup. The source-to-axis (SAD) distance is labeled as $D$. To install the ionisation chamber, the detector insert (grey) is replaced with a $2 \mathrm{~cm}$ thick Solid Water block containing a machined cavity for the ionisation chamber

\subsection{Ionisation chamber}

The ionisation chamber used in this study was a thimble type PTW Farmer ion chamber (Freiburg, Germany) of volume $0.6 \mathrm{~cm}^{3}$ and energy detection range of $30 \mathrm{kV}$ to $50 \mathrm{MV}$. The chamber was embedded to the geometric center of a Solid Water phantom block of $30 \times 30 \times 2$ $\mathrm{cm}^{3}$ with a specifically machined cavity for the chamber's sensitive volume which eliminates any air gap between the Solid Water and the detector. The ionisation chamber was used as the benchmark reference detector for the percentage depth dose measurements.

\subsection{Film}

The high-resolution mapping of the profiles was achieved with Ashland Gafchromic EBT3 film (Wayne, New Jersey). The film is self-developing, it is nearly tissue equivalent, and presents no orientation dependence [7]. Its spatial resolution depends on the optical scanner used to measure the optical density of the dose map; in this study the films were scanned with a Microtek ScanMaker i800 48-bit flatbed scanner, in transmission mode, using 72 dpi scanning resolution. The film needs at least 24 hours developing time to ensure a stable polymerisation to occur. The change in optical density (absorbance) of the film with respect to dose is not a linear 
relationship [6]; thus it is necessary to obtain a calibration curve in order to correctly reconstruct the measured dose as read by the film.

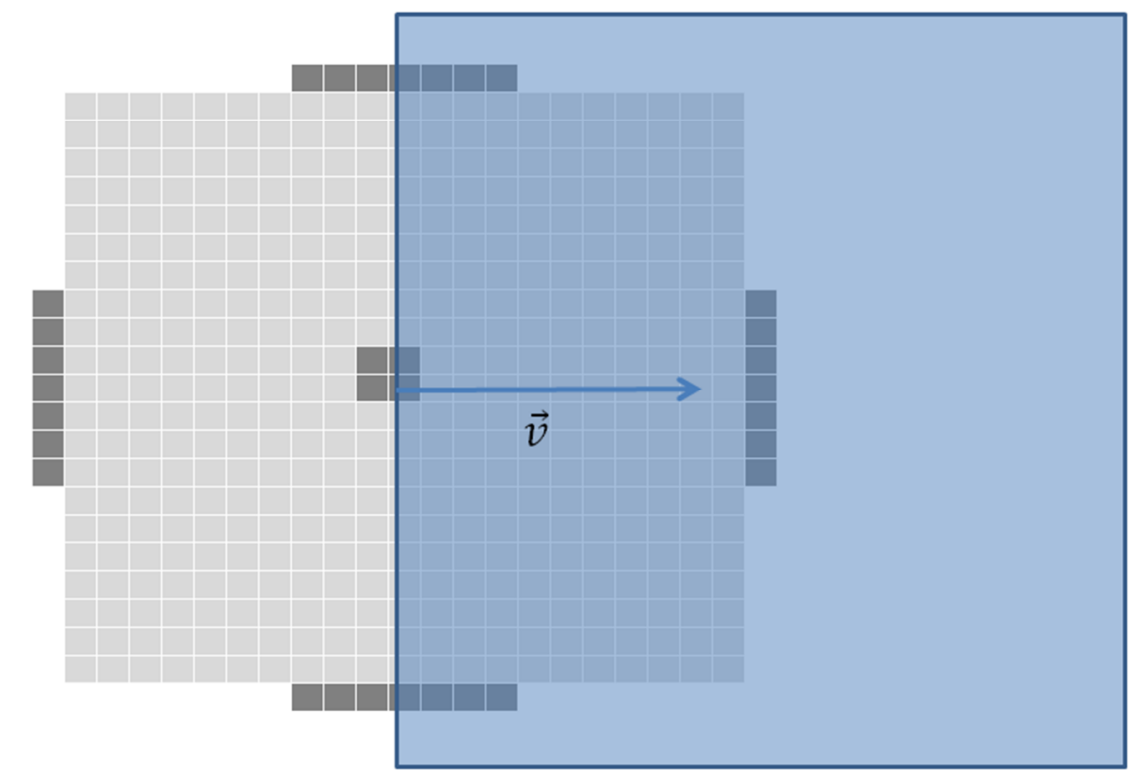

Figure 3: Diagram showing, in beam's eye view (BEV), the pixels of the MP512 (in dark grey) used for the timing study, and the shutter (blue) sweeping across the detector in the open sequence

\subsection{Dose calibration and equalization procedures}

Prior to any measurements, the dose rate of the gamma source was calculated using the ionisation chamber. The calculation accounted for temperature and pressure in the room and its purpose was to determine the length of irradiation to obtain a suitable signal-to-noise ratio for all the detectors. The calibrated ionisation chamber measurement of exposure $(\mathrm{X})$ was converted to dose rate $\left(\dot{D_{w}}\right)$ using the equation

$$
\dot{D_{w}}=\frac{X \cdot k_{T P} \cdot k_{D K} \cdot N_{w}}{t} \cdot 60\left(\frac{c G y}{\mathrm{~min}}\right)
$$

where $\mathrm{k}_{\mathrm{TP}}$ is the temperature and pressure correction factor, $\mathrm{k}_{\mathrm{DK}}$ is the decay correction factor, $\mathrm{N}_{\mathrm{w}}$ is the conversion factor from Roentgen to Gray $(0.967 \mathrm{cGy} / \mathrm{R})$, and $t$ is the duration of the acquisition in seconds. The same backscattering Solid Water slab of $6.5 \mathrm{~cm}$ was used for all the detectors with $24 \mathrm{~cm}$ source-to-axis distance (SAD), as shown in figure 2 .

The calibration factor of the MP512 was obtained by calculating the average charge per unit dose collected in the four central pixels of interest shown in figure 3 , at the same conditions used for the ionisation chamber measurements. Further, the MP512 consists of an array of pixels and the response of each pixel is different from its adjacent neighbors due to local substrate defects and parasitic capacitance associated with the connections' routing. The variation in preamplifier gain ranges within $0.1-0.5 \%$ of the dynamic range [10]. Thus the uniformity of the integral response of the diodes (pixels) could be slightly affected. Such nonuniformity can be corrected by irradiating the detectors with a flat field which was obtained by the means of optically stimulating the diode array with a homogeneous white light source (SMD LED) placed about 3 meters above the MP512 detector, thus obtaining an equalization factor for each pixel. The equalization factor was obtained by normalising the response of each 
individual channel to the flat field $\left(\mathrm{X}_{\mathrm{i}}\right)$ to the average response of all channels $\langle X\rangle$, thus generating the equalization factor $F_{i}$. To get the data equalized $X_{\text {eq-i }}$, the response of each pixel $Y_{i}$ is normalised by the equalisation factor $F_{i}[11]$ as shown by eq. 2.2 below.

$$
\mathrm{F}_{\mathrm{i}}=\frac{\mathrm{X}_{\mathrm{i}}}{\langle X>}, \quad \mathrm{X}_{\mathrm{eq}-\mathrm{i}}=\frac{\mathrm{Y}_{\mathrm{i}}}{\mathrm{F}_{\mathrm{i}}}
$$

The equalisation factors were applied to all of the MP512 detector datasets. A detailed study of the detector's uniformity is performed in ref. [8].

The optical density (OD) to dose calibration curve for the EBT3 film was obtained using eight squared samples of $3 \times 3 \mathrm{~cm}^{2}$ from the same sheet. Each sample was irradiated with a different dose, as determined using the calibrated ionisation chamber, ranging from $50 \mathrm{cGy}$ to 500 cGy. The film cut-outs were pre-scanned six times to ensure proper scanner warm-up and only the last three scans were used for analysis. The last three scans were split in their RGB channels using ImageJ (v.1.4.3.67) and only the red component of the scanned images was used. The scans were averaged and a region of interest within each film was used to obtain the optical density prior to irradiation. The same process was applied to the film sheets used to measure the beam profiles as described in section 2.8. Once the samples underwent irradiation, they were allowed to develop for 48 hours, after which the scanning procedure was repeated, again discarding the first three images to allow for scanner warm-up. The OD was then plotted against dose and a $3^{\text {rd }}$ order polynomial fitting curve was obtained, shown in figure 6 . Error evaluation was determined from the confidence limits in the conversion from OD to dose. The procedure is described in detail in ref. [8]. The average uncertainty across the film profiles is $\pm 2 \%$.

\subsection{Percentage depth dose}

The PDD measurements obtained by the ionisation chamber and MP512 have been compared. The SAD was set to be $24 \mathrm{~cm}$ (figure 2). This distance was chosen to be the typical distance where the animals or the sample flasks will be placed for irradiation, and was kept constant throughout the measurements. The ionisation chamber was placed in the $2 \mathrm{~cm}$ thick Solid Water insert. Backscattering Solid Water $6.5 \mathrm{~cm}$ thick was used. The measurements were performed by increasing $d$ (figure 2) in increments of 5 and $10 \mathrm{~mm}$, from $10 \mathrm{~mm}$ up to $50 \mathrm{~mm}$ with the shortest possible pauses between measurements, to minimise the detectors' temperature dependence and the ionisation chamber's atmospheric pressure dependence. The measurements were taken using an open field of $10 \times 10 \mathrm{~cm}^{2}$ without any collimators in the beam, to maximise lateral electronic equilibrium. Duration of ionisation chamber measurements was set to 100 seconds each, while MP512 measurement duration was set to 20 seconds. The PDD results are shown in figure 5.

\subsection{Beam output factors}

The output factor of a photon beam (OF) is a parameter that is defined as "the ratio of dose per monitor unit at a specific field size to the reference field size" [8],[12]. Especially in small beams where lateral electronic equilibrium is not fully established, it is important to determine the output factor. The output factors of the beams were obtained using film and MP512 detectors. A region of interest in the center of each beam was used to determine the average dose. Areas of $5 \times 5 \mathrm{~mm}^{2} ; 4 \times 4 \mathrm{~mm}^{2}$; and $2.5 \times 2.5 \mathrm{~mm}^{2}$ were chosen as regions of interest in the film dose maps for the $20 \mathrm{~mm}, 10 \mathrm{~mm}$ and $5 \mathrm{~mm}$ beams respectively. In the case of MP512, four central pixels were used for the two larger beams, while two pixels were used in the case of the $5 \mathrm{~mm}$ beam. The response of the pixels was averaged, then normalised to the $10 \times 10 \mathrm{~cm}^{2}$ open beam; the results are shown in figure 8 . 


\subsection{Beam profiles}

The measurement of the beam profiles was performed using the MP512 detector and radiochromic film, for each collimator, at depth $1.5 \mathrm{~cm}$ and SAD $24 \mathrm{~cm}$. The duration of MP512 measurements was 40 seconds (twice the duration used for PDD study to account for smaller beam OF and thus to increase SNR). Three measurements were performed for each MP512 profile and averaged. Horizontal \& vertical profiles were generated from the center of each beam.

Film measurements were performed using $6 \times 6 \mathrm{~cm}^{2}$ film samples. Based on the output factors, each film sample was irradiated for a period of time corresponding to a 200-300 cGy dose in the center of the field, in order to obtain good SNR. Penumbra (the edge of a profile corresponding to a sharp variation in dose between 20\% and 80\%) and FWHM were obtained using MATLAB curve fitting tool (shape-preserving) and compared for the two dosimeters. The profiles are shown in figure 7 and tabulated in table 1.

\subsection{Shutter timing study}

The time it takes for the shutter to fully open (ramp-up) and close (ramp-down) has been investigated to evaluate the effect of the transient on the total dose delivered. The SAD was 24 $\mathrm{cm}$, depth of detector $1.5 \mathrm{~cm}$, and $10 \times 10 \mathrm{~cm}^{2}$ equivalent field (no collimator) was used. The scheme of the measurement setup can be seen in figure 3. Figure 4 shows the schematic diagram of the normalized response of pixels as a function of time. A raising edge is defined as the time interval between $5 \%$ and $95 \%$ of the normalised transient, depicted as $\Delta \mathrm{t}_{1}$. A falling edge is defined as the interval between $95 \%$ and $5 \%$ of the response, depicted as $\Delta \mathrm{t}_{2}$. Knowing the average dose rate from the cobalt-60 source, the delivered dose during the transients can be calculated; in this study, the doses associated with the raising edge and falling edge are defined as $D_{t 1}$ and $D_{t 2}$, respectively. The seven pixels from each edge and four pixels from the center (figure 3) were chosen to analyse the timing of the shutter. Beam-on duration was set to 20 seconds. The response of a central pixel as a function of time is shown in figure 9.

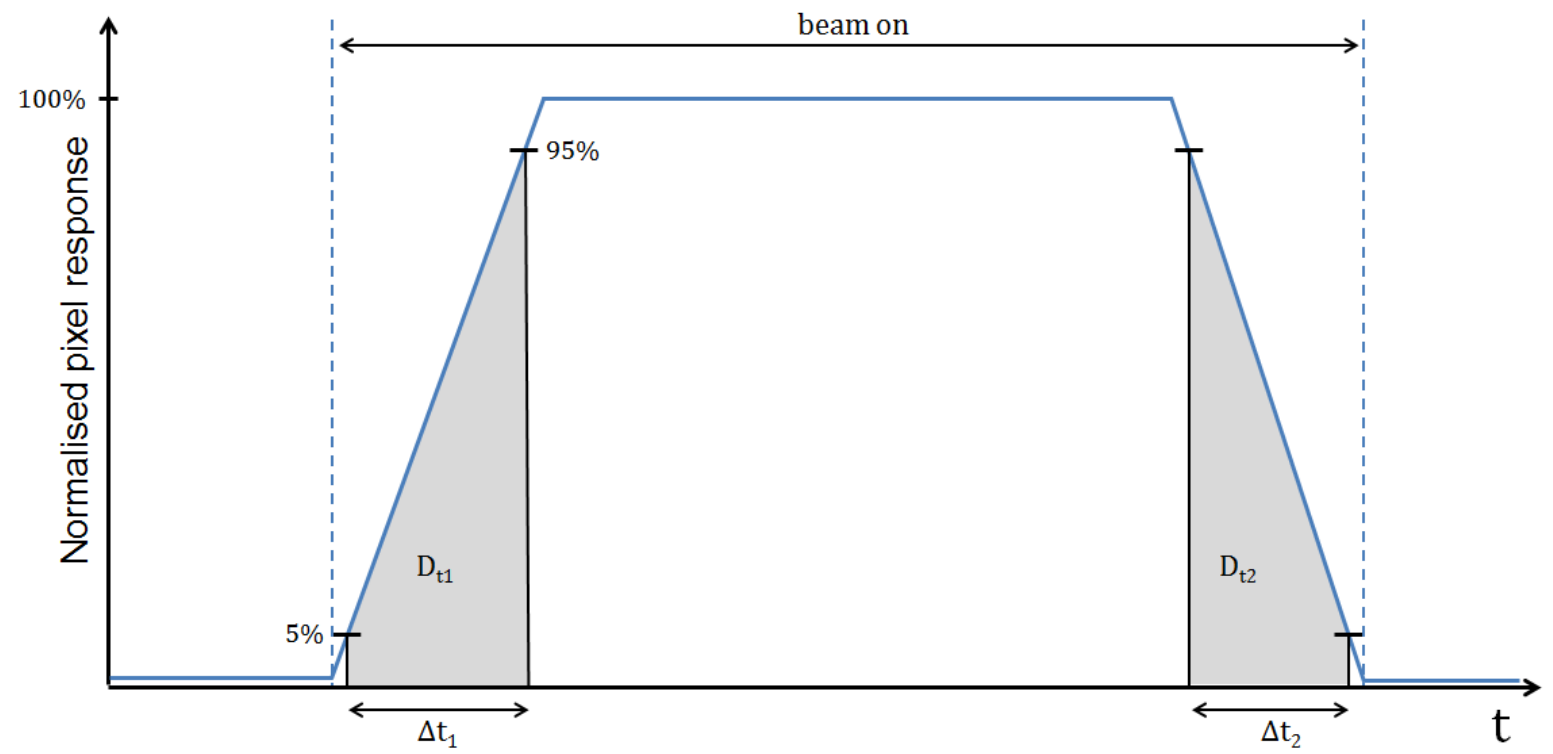

Figure 4: Diagram outlying the ramp-up and ramp-down transients in the response of the pixels, as a function of time 


\section{Results and discussion}

The radiation source of the Co-60 irradiator emits gamma rays continuously with an average dose rate which was determined to be $(10.65 \pm 0.03) \mathrm{cGy} / \mathrm{min}$ at $1.5 \mathrm{~cm}$ depth in Solid Water, using the ionisation chamber. Compared to the instantaneous dose rate of a clinical linac that ranges from 0.5 to $6 \mathrm{~Gy} / \mathrm{min}$ at the same depth, it is clear that the dose rate of the Theratron source is very low. While the usual application of the MP512 detector and readout system is optimized for use in clinical linac quality assurance, in order to maximise the SNR in this specific application, MP512 has been set with the third highest sensitivity available $(0.5 \mathrm{pC}$ full dynamic range) and the longest integration time available (2 ms) assessing the uncertainty associated with MP512 measurements within a maximum of $\pm 3 \%$ (two standard deviations) for repeated measurements. The calibration factor of the MP512 was calculated to be $242.5 \pm 0.03$ $\mathrm{pC} / \mathrm{cGy}$.

\subsection{Percentage depth dose}
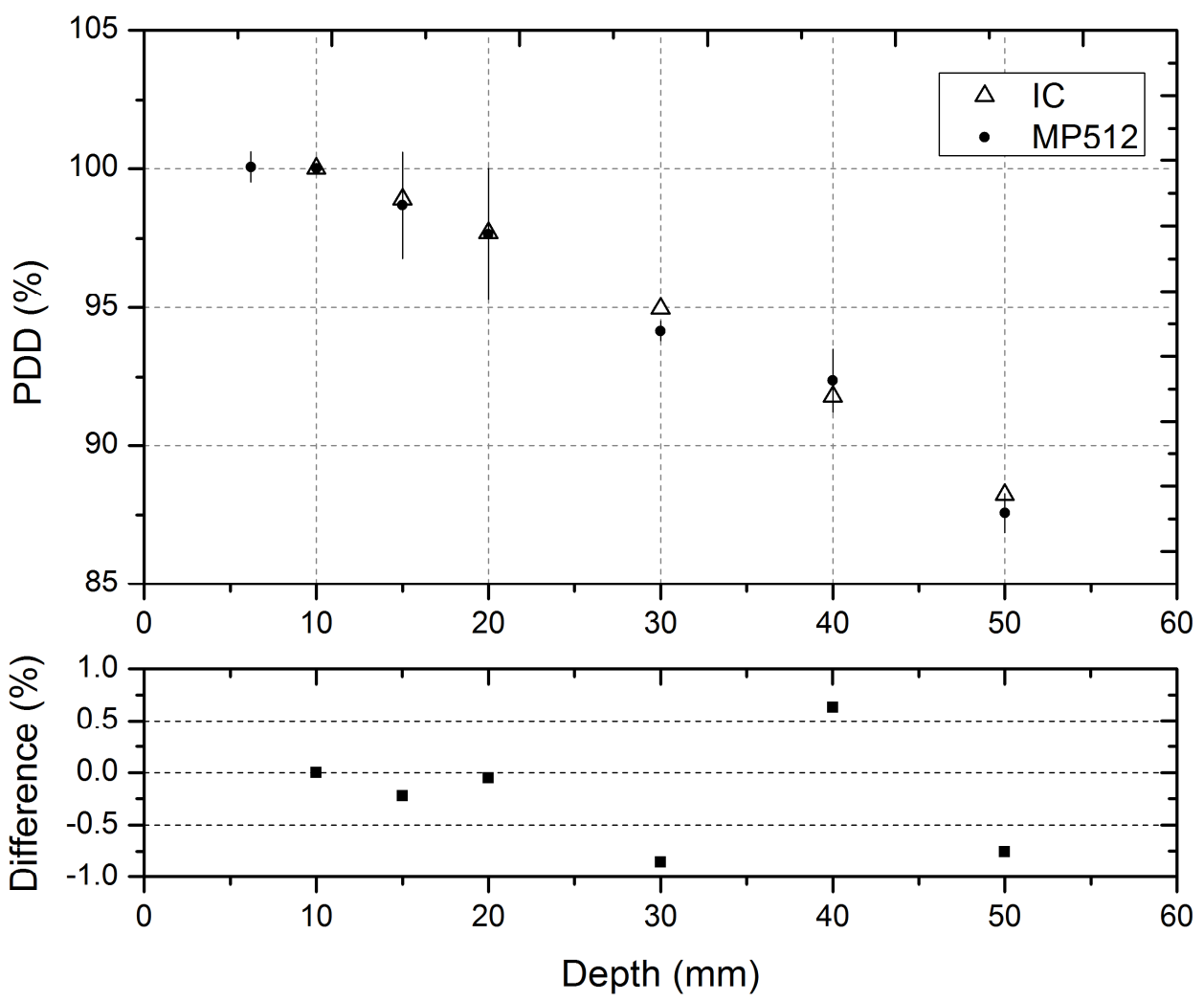

Figure 5: Percentage depth dose response for ionization chamber and MagicPlate-512 detector. The percentage difference is shown between the two dosimeters.

The comparison between ionisation chamber and MP512 percentage depth dose response is shown in figure 5. Ion chamber measurements had negligible variation between the two measurements for each depth, with the remaining uncertainty being only the limitation of the electrometer's smallest increment of $0.2 \%$. The variation between PDD measurements taken with MP512 detector ranged from $0.36 \%$ to $2.4 \%$ between three repeated measurements for every depth. Despite the variation in the MP512 response, the discrepancy between MP512 and ionisation chamber percentage depth dose measurements is within $1 \%$. 


\subsection{Beam profiles}
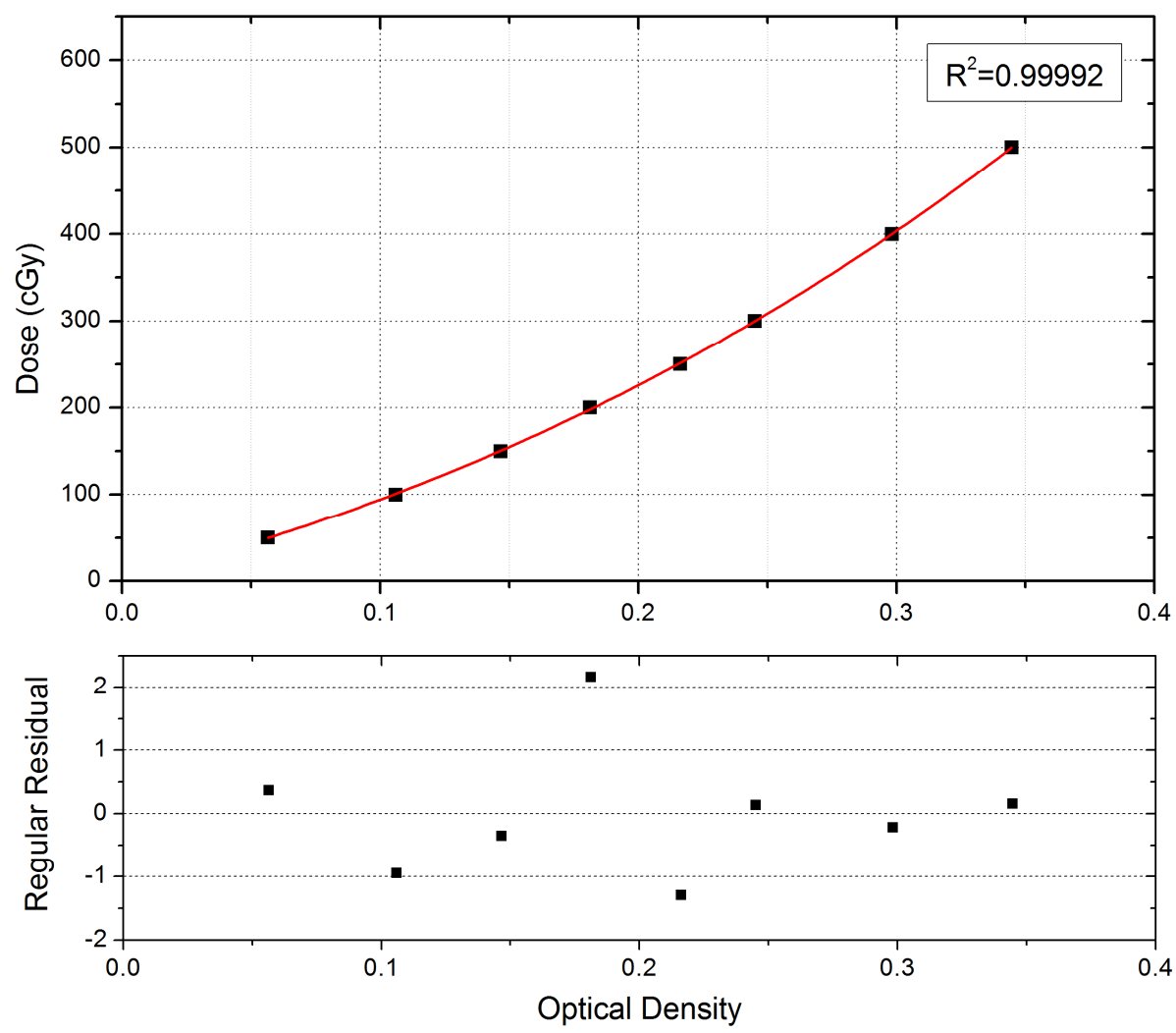

Figure 6: Calibration curve for EBT3 film

Film samples received a dose ranging from $230 \mathrm{cGy}$ to $300 \mathrm{cGy}$, based on the source's dose rate as determined in section 2.5. The beam profiles in the horizontal and vertical axis of the BEV are shown in figure 7, and the FWHM and 20\%-80\% penumbra values are summarised in table 1 . The horizontal and vertical profiles taken from the $2 \mathrm{D}$ dose maps show a symmetric dose buildup and drop-off for the $20 \mathrm{~mm}$ and $10 \mathrm{~mm}$ collimators. Of particular interest is the 5 $\mathrm{mm}$ beam profiles and the evident asymmetry of the dose distribution. A 3D representation of the $5 \mathrm{~mm}$ beam profile measured by film is presented in figure $7(\mathrm{~g})$ to better visualise the asymmetry. Besides the reduced lateral electronic equilibrium, this small beam is also affected by irregularities present in the collimator due to its machining, and is thus asymmetric. The differences in penumbra and FWHM between film and MagicPlate detector are lower than 0.9 $\mathrm{mm}$ showing the suitability of MP512 to perform the quality check of the beam profiles in realtime prior to animal or cell samples irradiations. Spatial resolution of the EBT3 film is limited by the capabilities of the optical scanner and the uncertainty in the optical density curve fitting. 


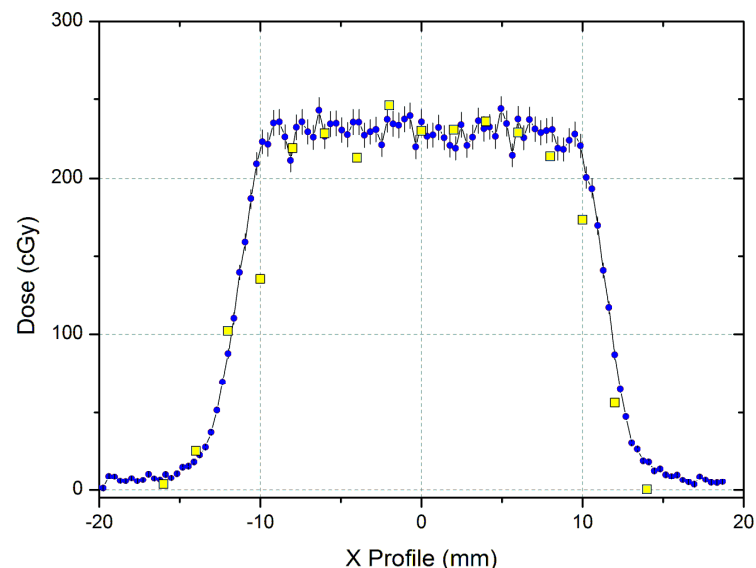

(a)

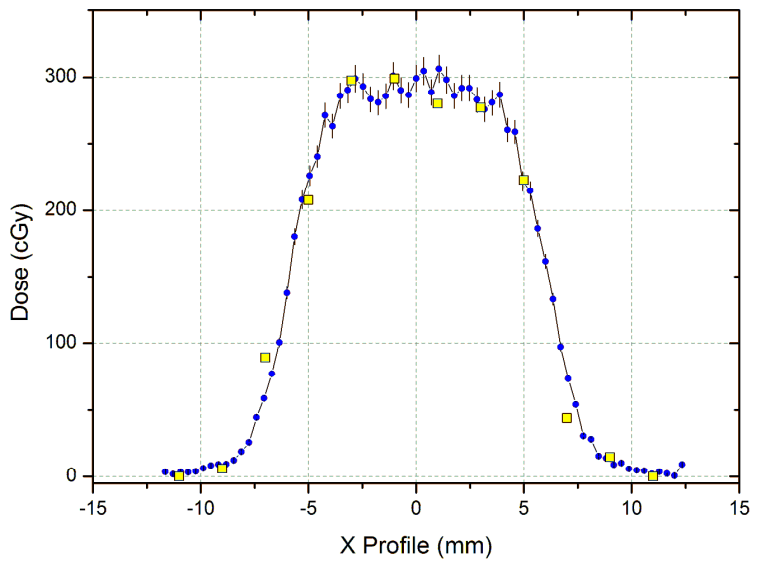

(c)

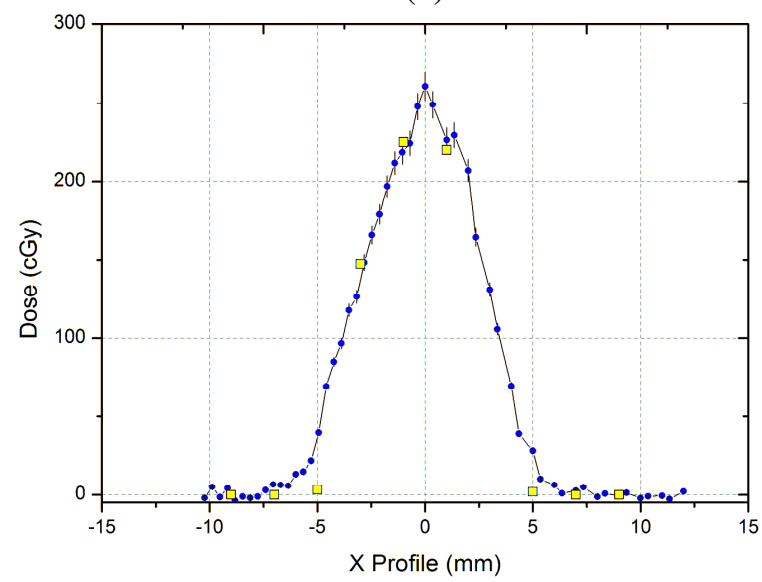

(e)

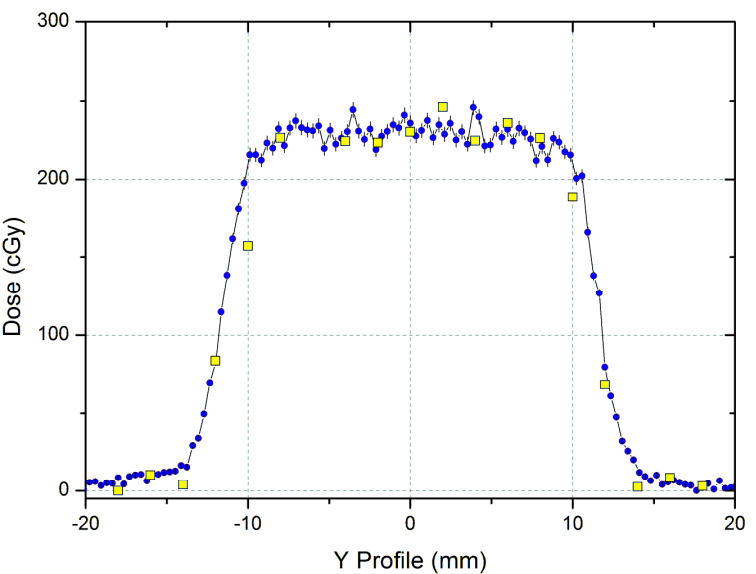

(b)

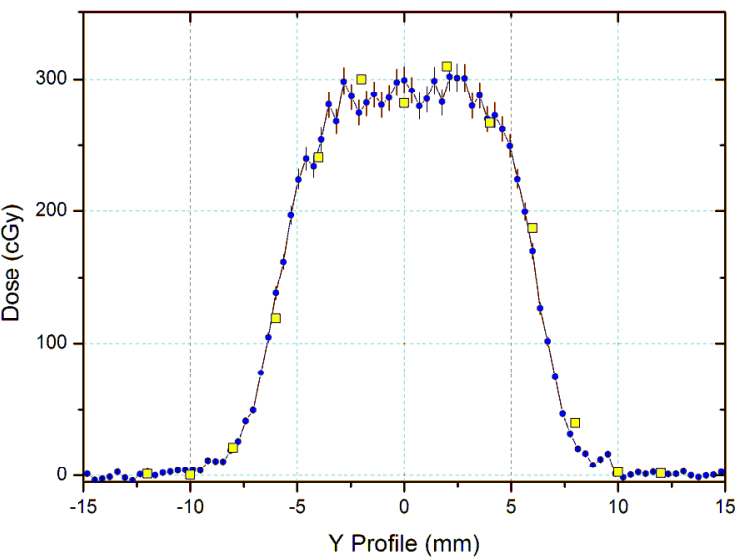

(d)

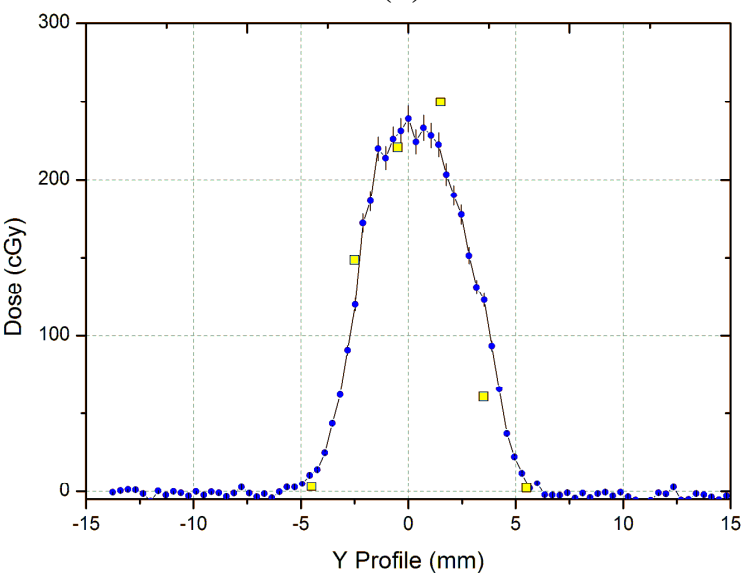

(f) 


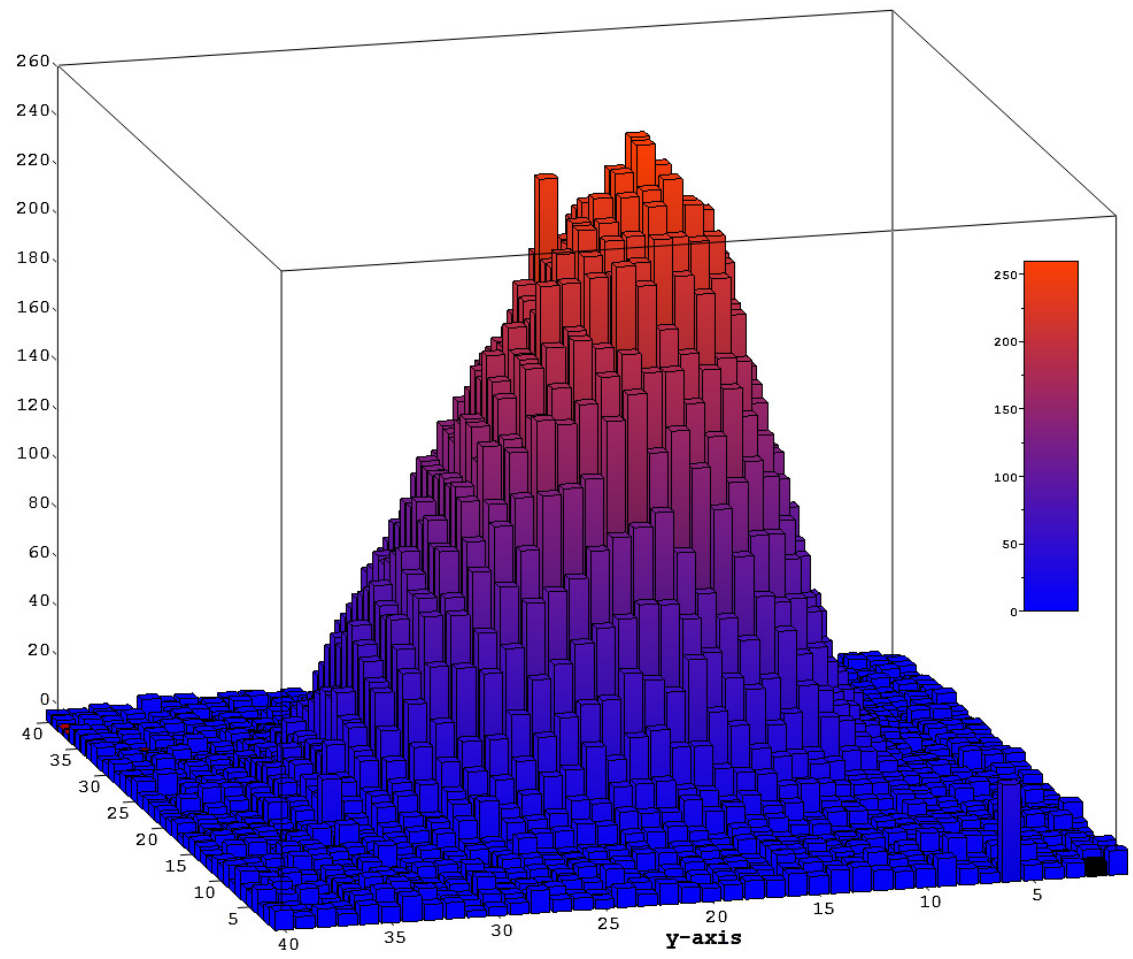

(g)

Figure 7: Dose profile measurements for (row 1) $20 \mathrm{~mm}$; (row 2) $10 \mathrm{~mm}$; and (row 3 ) $5 \mathrm{~mm}$ beams using radiochromic film (blue) and MP512 (yellow); (bottom) asymmetry of the $5 \mathrm{~mm}$ profile shown in 3D. Error bars are due to the uncertainties in film response and alignment.

Table 1: Comparison of FWHM and 20\%-80\% penumbra for EBT3 film and MagicPlate512

\begin{tabular}{|llllllll|}
\hline & $\begin{array}{l}\text { Beam } \\
\text { Diameter } \\
(\mathrm{mm})\end{array}$ & $\begin{array}{l}\text { Horizontal } \\
\text { Film }\end{array}$ & MP512 & $\begin{array}{l}\text { Difference } \\
(\mathrm{mm})\end{array}$ & $\begin{array}{l}\text { Vertical } \\
\text { Film }\end{array}$ & MP512 & $\begin{array}{l}\text { Difference } \\
(\mathrm{mm})\end{array}$ \\
\hline \multirow{2}{*}{ FWHM } & 20 & 23.172 & 22.282 & 0.89 & 23.333 & 22.405 & 0.928 \\
& 10 & 12.05 & 12.035 & 0.015 & 11.98 & 12.02 & -0.04 \\
& 5 & 5.218 & 5.637 & -0.419 & 6.062 & 5.751 & 0.311 \\
\hline \multirow{3}{*}{ Penumbra } & 20 & 2.017 & 2.655 & -0.638 & 1.954 & 2.365 & -0.411 \\
& 10 & 2.527 & 1.763 & 0.764 & 2.13 & 2.775 & -0.645 \\
& 5 & 1.606 & 0.862 & 0.744 & 2.358 & 1.507 & 0.851 \\
\hline
\end{tabular}

\subsection{Beam output factors}

The output factors for the different pencil beams are shown in figure 8. The uncertainties in the graph are due to the statistical variation in the film response and low SNR due to the very low dose rate delivered by the Co-60 irradiator. The error bars in the $5 \mathrm{~mm}$ data point are larger due to the asymmetry of the dose profile which affects the average dose calculated across the region of interest. The MP512 and film results agree within one standard deviation. 


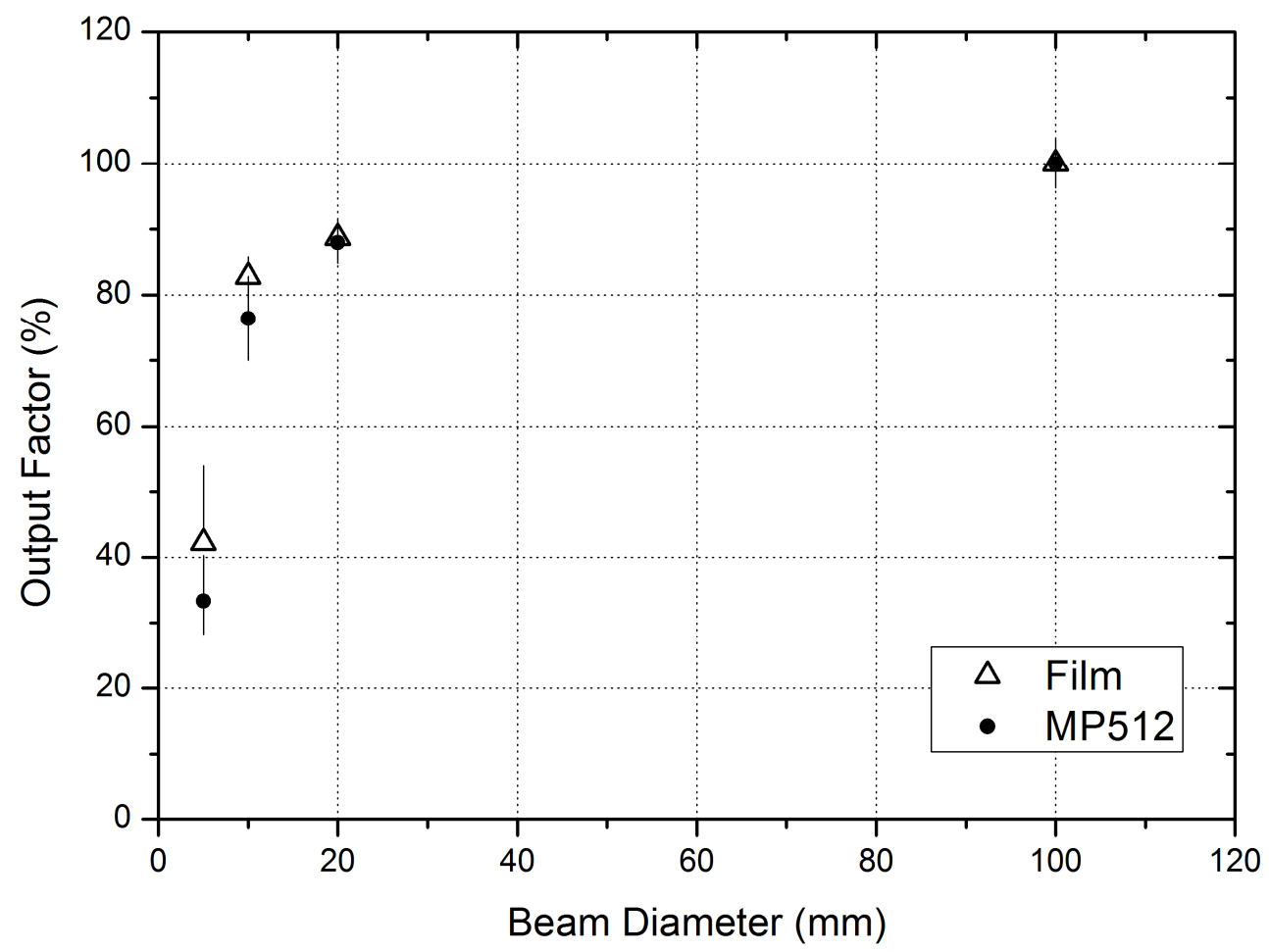

Figure 8: Output factors for EBT3 film and MagicPlate-512

\subsection{Shutter timing study}

The ramp-up and ramp-down transient study has been performed with the Romulus data analysis toolkit. When the shutter is opened, the pixels on the left started 'seeing' the source before the pixels on the right (figure 3). The duration of the shutter reaching the right quadrant was about $(250 \pm 10) \mathrm{ms}$. There was no delay between the top and bottom quadrants 'seeing' the source, as the pixels were exposed to the beam at the same time in the vertical direction. For the central pixels of interest, $\Delta \mathrm{t}_{1}$ was found to be $(151 \pm 15) \mathrm{ms}$, and $\Delta \mathrm{t}_{2}$ was $(165 \pm 15) \mathrm{ms}$. $\mathrm{D}_{\mathrm{t} 1}$ was calculated to be $\left(1.23 \times 10^{-2} \pm 0.05 \times 10^{-2}\right) \mathrm{cGy}$, and $\mathrm{D}_{\mathrm{t} 2}$ was calculated to be $\left(1.37 \times 10^{-2} \pm 0.05\right.$ $\left.\times 10^{-2}\right) \mathrm{cGy}$, corresponding to a total transient dose of $\left(2.60 \times 10^{-2} \pm 0.07 \times 10^{-2}\right) \mathrm{cGy}$. The transient dose becomes non-negligible for small-dose irradiations (less than $1 \mathrm{cGy}$ ) and should be taken into account.

When the beam is turned on, the shutter exposes the cobalt source at a constant speed of about $20 \pm 2 \mathrm{~cm} / \mathrm{s}$. It was observed that there was a variation of the $\Delta \mathrm{t}_{2}$ parameter across the pixels in the horizontal direction, in the form of a residual tail response as a function of time for the pixels in the left quadrant, when compared to pixels in the right quadrant, indicating that the shutter's speed is not constant when closing and concealing the source. It was found that in the first $50 \pm 5 \mathrm{~ms}$ of the shutter beginning to close, the shutter covers about $50 \%$ of the MP512 detector's pixels, from right to left, corresponding to a shutter speed of around $55 \mathrm{~cm} / \mathrm{s}$. About $75 \%$ of the detector is covered in $250 \pm 20 \mathrm{~ms}$; at this stage, the shutter slows down to 16.5 $\mathrm{cm} / \mathrm{s}$. The remaining $25 \%$ in the far left quadrant of the detector takes up to $750 \mathrm{~ms}$ to be fully shielded by the shutter from the moment it begins to close. The average shutter closing speed was calculated to be $(26 \pm 4) \mathrm{cm} / \mathrm{s}$. Thus, the shutter will traverse the $20 \mathrm{~mm}$ pencil beam in about $77 \mathrm{~ms}$, and the $5 \mathrm{~mm}$ beam in $19 \mathrm{~ms}$. Uncertainties in these results were due to the 
statistical fluctuations of the detector response. These were minimised by removing the noise baseline of the pixels of interest, if present.

The variation in the shutter speed as it closes is due to the shutter's transport mechanism. By default, the shutter is kept closed by a spring. When opening, a motor drives the shutter towards the spring, leading to a constant speed as observed by the MP512 detector; the motor actively keeps the shutter in the open position. To close the shutter, the power to the motor is temporarily cut off, leading to the spring relaxing and moving the shutter to conceal the source. About halfway through the movement, the motor re-engages to prevent the shutter from slamming and induce damage to the mechanical transport system. This 'active open' system ensures that even in the event of power loss, the source is automatically shielded by the springacted shutter.
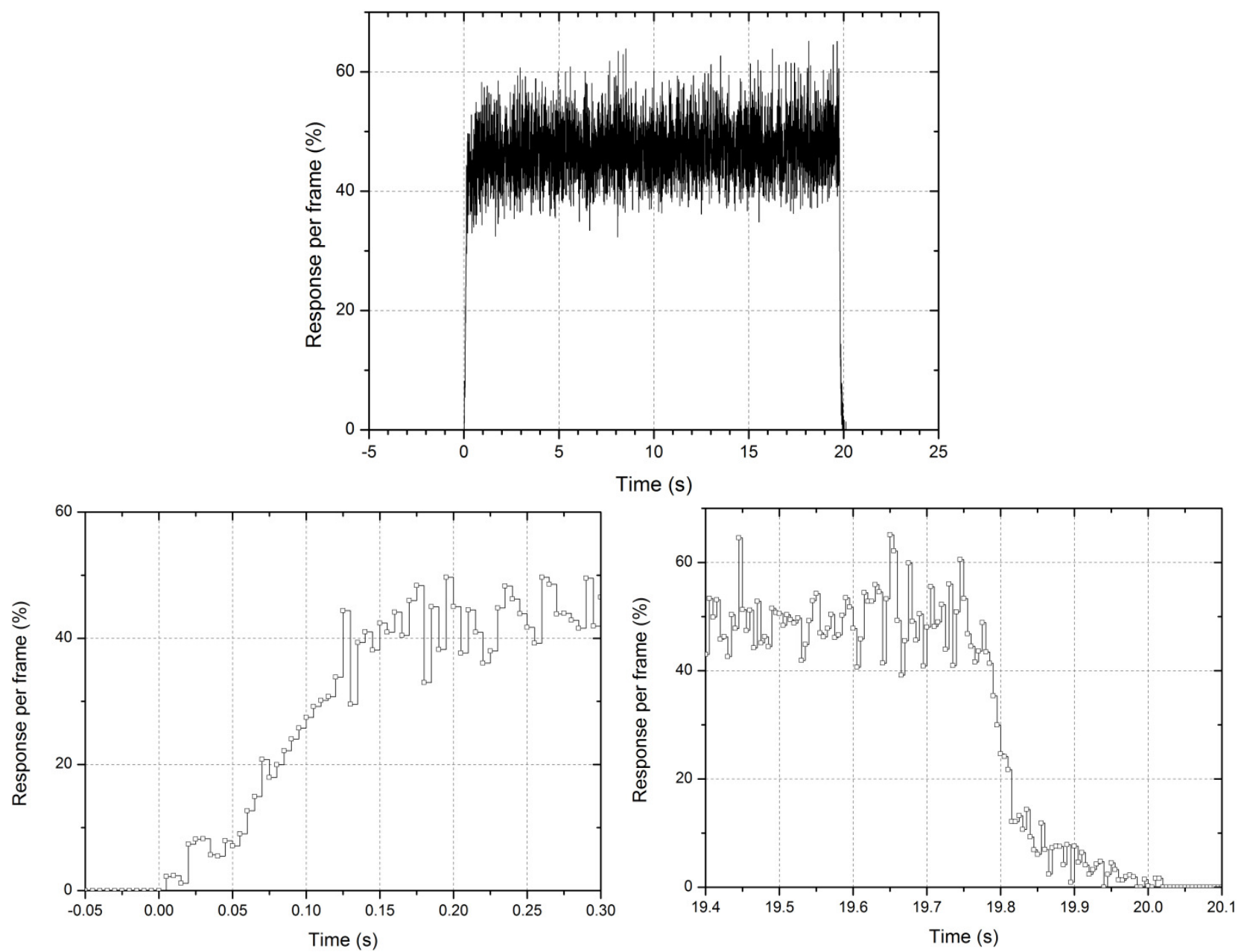

Figure 9: (top) Response of one pixel of interest from the center of MP512, chosen arbitrarily out of the four. The response is shown as a percentage of the dynamic range $(0.5 \mathrm{pC})$; (bottom) expanded view of the transients

\section{Conclusion}

In this study, the characteristics of a Theratron Co-60 source for small animal and cell irradiation studies were investigated using an ionisation chamber, radiochromic EBT3 film and a silicon detector array system, named MagicPlate-512. Dose rate of the Co-60 source was 
found to be $(10.65 \pm 0.03) \mathrm{cGy} / \mathrm{min}$, and the MP512 calibration factor was calculated to be $(242.5 \pm 0.03) \mathrm{pC} / \mathrm{cGy}$ at $1.5 \mathrm{~cm}$ depth in Solid Water. Percentage depth dose measurements taken with ionisation chamber and MP512 agree within $1 \%$ up to a depth of $50 \mathrm{~mm}$ in Solid Water. It was found that the $20 \mathrm{~mm}$ and $10 \mathrm{~mm}$ diameter beams have uniform penumbra regions. The $5 \mathrm{~mm}$ beam showed a significant asymmetric dose profile due to irregular machining of the lead collimator. Full-width at half-maximum studies and 20\%-80\% penumbra evaluations performed with EBT3 film and MP512 agree within less than $1 \mathrm{~mm}$. Output factors obtained with film and MagicPlate detector agree within 3\% for all three collimators. MagicPlate-512 has been used to characterise the radiation source's shutter opening using its superior temporal resolution and sensitivity. The shutter speed of the container was found to be an average of about $20 \mathrm{~cm} / \mathrm{s}$ when opening and $26 \mathrm{~cm} / \mathrm{s}$ when closing. Despite the low source activity, the MagicPlate-512 dosimetry system has agreed within small uncertainties in comparison with ionisation chamber and EBT3 film proving it is suitable for real-time verification of very low dose rate irradiators, providing high spatial and temporal resolution.

\section{References}

[1] B.J. Blyth, S. Kakinuma, M. Sunaoshi, Y. Amasaki, S. Hirano-Sakairi, K. Ogawa, A. Shirakami, Y. Shang, C. Tsuruoka, M. Nishimura, Y. Shimada, Genetic analysis of T cell lymphomas in carbon ion-irradiated mice reveals frequent interstitial chromosome deletions: implications for second cancer induction in normal tissues during carbon ion radiotherapy, PLoS ONE, 10(6), 2015, pp 124.

[2] A.R. Pinnapureddy, C. Stayner, J. McEwan, O. Baddeley, J. Forman, M.R. Eccles, Large animal models of rare genetic disorders: sheep as phenotypically relevant models of human genetic disease, Orphanet Journal of Rare Diseases, 10(107), 2015, pp 1-8.

[3] T. Zhang, L. Zhang, S. Fan, M. Zhang, H. Fu, Y. Liu, et al., Patient-derived gastric carcinoma xenograft mouse models faithfully represent human tumor molecular diversity, PLoS ONE, 10(7), 2015, pp 1-13.

[4] H.Y. Yoo, A. Zeifman, E.A. Ko, K.A. Smith, J. Chen, R.F. Machado, Y. Zhao, R.D. Minshall, J.X.J. Yuan, Optimization of isolated perfused/ventilated mouse lung to study hypoxic pulmonary vasoconstriction, Pulmonary Circulation, 3(2), 2013, pp 396-405.

[5] International Atomic Energy Agency (IAEA), Absorbed dose determination in external beam radiotherapy: an international code of practice for dosimetry based on standards of absorbed dose to water, Technical Reports Series, No. 398, 2000

[6] M.J. Butson, P.K.N. Yu, T. Cheung, P. Metcalfe, Radiochromic film for medical radiation dosimetry, Materials Science and Engineering, R 41, 2003, pp 61-120.

[7] V.C. Borca, M. Pasquino, G. Russo, P. Grosso, D. Cante, P. Sciacero, G. Girelli, M.R. La Porta, S. Tofani, Dosimetric characterization and use of GAFCHROMIC EBT3 film for IMRT dose verification, Journal of Applied Clinical Medical Physics, 14(2), 2013, pp 158-171.

[8] A.H. Aldosari, M. Petasecca, A. Espinoza, M. Newall, I. Fuduli, C. Porumb, S. Alshaikh, Z.A. Alrowaili, M. Weaver, P. Metcalfe, M. Carolan, M.L.F. Lerch, V. Perevertaylo, A.B. Rosenfeld, A two dimensional silicon detectors array for quality assurance in stereotactic radiotherapy: MagicPlate-512, Med. Phys., 41(9), 2014, pp 091707 1-10. 
[9] I. Fuduli, C. Porumb, A. Espinoza, A. Aldosari, M. Carolan, M.L.F Lerch, P.E. Metcalfe, A. Rosenfeld, M. Petasecca, A comparative analysis of multichannel data acquisition systems for quality assurance in external beam radiation therapy, JINST, 9(6), 2014, pp 1-12.

[10] http://www.ti.com/lit/gpn/afe0064, www.ti.com [Online], Texas Instruments, 64 channel analog front end for digital $x$-ray detector, 2009.

[11] A. Bocci, M.A. Cortés-Giraldo, M.I. Gallardo, J.M. Espino, R. Arráns, M.A.G. Alvarez, Z. AbouHaïdar, J.M. Quesada, A. Pérez Vega-Leal, F.J. Pérez Nieto, Silicon strip detector for a novel $2 D$ dosimetric method for radiotherapy treatment verification, Nuclear Instruments and Methods in Physics Research Section A: Accelerators, Spectrometers, Detectors and Associated Equipment, vol. 673, 2012, pp 98-106.

[12] E.B. Podgorsak, Radiation oncology physics: a handbook for teachers and students, IAEA, 2005. 